\title{
Comparison of Gamma Pass Rate between the Dose-to-Water and Dose-to-Medium Reporting Modes for Patient-Specific QA Using a Helical Diode Array Dosimeter with a Fixed Phantom Density
}

\author{
Hideaki Hirashima1, Mitsuhiro Nakamura, ${ }^{1 *}$, Yoshitomo Ishihara', Nobutaka Mukumoto1, \\ Mami Akimoto1, Tsuneyuki Tomita ${ }^{3}$, Yoshinori Hirose ${ }^{3}$, Kenji Kitsuda ${ }^{3}$, Takashi Ishigaki ${ }^{4}$, \\ Takashi Mizowaki1
}

\footnotetext{
${ }^{1}$ Department of Radiation Oncology and Image-Applied Therapy, Graduate School of Medicine, Kyoto University, Kyoto, Japan ${ }^{2}$ Department of Information Technology and Medical Engineering, Faculty of Human Health Science, Graduate School of Medicine, Kyoto University, Kyoto, Japan

${ }^{3}$ Division of Radiology, Osaka Red Cross Hospital, Osaka, Japan

${ }^{4}$ Department of Radiation Therapy, Osaka Red Cross Hospital, Osaka, Japan

Email: ${ }^{\star}$ m_nkmr@kuhp.kyoto-u.ac.jp
}

\begin{abstract}
How to cite this paper: Hirashima, H., Nakamura, M., Ishihara, Y., Mukumoto, N., Akimoto, M., Tomita, T., Hirose, Y., Kitsuda, K., Ishigaki, T. and Mizowaki, T. (2018) Comparison of Gamma Pass Rate between the Dose-to-Water and Dose-toMedium Reporting Modes for PatientSpecific QA Using a Helical Diode Array Dosimeter with a Fixed Phantom Density. International Journal of Medical Physics, Clinical Engineering and Radiation Onco$\log y, 7,74-86$.
\end{abstract}

https://doi.org/10.4236/ijmpcero.2018.71007

Received: January 11, 2018

Accepted: February 20, 2018

Published: February 23, 2018

Copyright ( 92018 by authors and Scientific Research Publishing Inc. This work is licensed under the Creative Commons Attribution-NonCommercial International License (CC BY-NC 4.0). http://creativecommons.org/licenses/by-nc/4.0/ cc) (i) (9) Open Access

\begin{abstract}
Introduction: To compare the measured dose distributions to calculated ones in dose-to-water $\left(D_{w}\right)$ and dose-to-medium $\left(D_{m}\right)$ reporting modes for simple plans and patient-specific intensity modulated radiation therapy (IMRT) and volumetric modulated arc therapy (VMAT) plans using ArcCHECK with a fixed phantom density. Methods: The recommended density value of 1.18 $\mathrm{g} / \mathrm{cm}^{3}$ for Acuros XB and X-ray voxel Monte Carlo was assigned to ArcCHECK on CT images. A total of 45 simple plans, including a 1-field plan, a 3 -field plan, a 4 -field plan, a half-arc plan from $270^{\circ}$ to $90^{\circ}$, and a full-arc plan, were assessed. Subsequently, the patient-specific 96 IMRT and VMAT plans were evaluated. Gamma analysis with a 3\% normalized global dose error and a $3 \mathrm{~mm}$ distance-to-agreement criteria $(\gamma 3 \% \mathrm{G} / 3 \mathrm{~mm})$ was performed in the $D_{w}$ and $D_{m}$. The change in $\gamma 3 \% \mathrm{G} / 3 \mathrm{~mm}$ between $D_{w}$ and $D_{m}$ were statistically analyzed using JMPPro11 software. Results: The median values of $\gamma 3 \% \mathrm{G} / 3 \mathrm{~mm}$ for all simple plans for $D_{w}$ and $D_{m}$ were $98.1 \%$ (range, $75.2 \%$ $100 \%$ ) and $95.5 \%$ (range, $23.7 \%-100 \%$ ), respectively ( $\mathrm{p}<0.01$ ). In the patient-specific IMRT and VMAT plans, the median values of $\gamma 3 \% \mathrm{G} / 3 \mathrm{~mm}$ for $D_{w}$ and $D_{m}$ were $98.6 \%$ (range, $90.1 \%-100 \%$ ) and $90.5 \%$ (range, $38.5 \%$ 97.2\%), respectively $(\mathrm{p}<0.01)$. Conclusion: Our results showed that the cal-
\end{abstract}


culated and measured dose distributions were in good agreement for $D_{w}$, but were not for $D_{m}$. From the viewpoint of the rationale of dosimetry, $D_{w}$ shows better agreement with measured dose distribution when using the fixedphantom density recommended by the vendor.

\section{Keywords}

Helical Diode Array Dosimeter, Dose-to-Water, Dose-to-Medium, Gamma Pass Rate

\section{Introduction}

Worldwide, intensity modulated radiation therapy (IMRT) and volumetric modulated arc therapy (VMAT) are routinely used in clinical practice. These techniques have improved patient outcomes compared with three-dimensional (3D) conformal radiotherapy [1] [2].

The multi-leaf collimator (MLC), gantry position, and/or dose rate vary continuously during IMRT and VMAT delivery. Such complicated beam delivery techniques lead to dosimetric differences between measurements and calculations; therefore, patient-specific quality assurance (QA) is required to validate the calculated dose distribution [3]. Patient-specific QA procedures differ from traditional approaches using an ionization chamber and films to two-dimensional (2D) planar or 3D volumetric dosimeters. One of the advantages of 3D volumetric dosimetry over 2D planar dosimetry is that volumetric dose distributions can be assessed with a single measurement.

Dose calculation algorithms have evolved dramatically from measurementbased to model-based, linear Boltzmann transport equation (LBTE)-based, and Monte Carlo (MC)-based methods. Model-based algorithms, such as superposition/convolution algorithms, anisotropic analytic algorithms (AAA), and collapsed cone convolution/superposition (CCCS), provide more accurate results in low-density areas than measurement-based algorithms. The model-based calculation algorithm computes transport and dose deposition using radiological and density scaling deposited by the absorbed dose in water. LBTE- and MC-based dose calculation algorithms, such as Acuros XB (AXB) and X-ray voxel Monte Carlo (XVMC), respectively, are more accurate in heterogeneous regions than model-based dose calculation algorithms [4]-[16]. As a feature of the AXB and XVMC, these algorithms can calculate different dose reporting modes in water [dose-to-water $\left.\left(D_{w}\right)\right]$ and in medium [dose-to-medium $\left.\left(D_{m}\right)\right]$ through the stopping power ratio of water-to-medium [8] [13] [17].

Recently, ArcCHECK (Sun Nuclear Corp., Melbourne, FL), which is one of the $3 \mathrm{D}$ volumetric dosimeters, has been clinically used as a patient-specific QA tool. Several studies on ArcCHECK have been reported with a focus on angular, directional, and field size dependencies, and response to scattering and leakage radiations in a fixed arc [18]-[24]. In these reports, patient-specific QA using 
ArcCHECK was also performed with AAA or CCCS algorithms [18]-[24]. Eaton et al. reported the comparison of measured dose distributions and calculated ones with several dose calculation algorithms, including MC-based method, by use of ArcCHECK. However, the report makes no mention of dose reporting modes in MC-based method [25]. The dose distribution between $D_{w}$ and $D_{m}$ was different in the "water-unlike" tissues with densities, due to the difference in stopping power ratio and fluence [8]. Taken together, a comparison between measured and calculated dose distributions in $D_{w}$ and $D_{m}$ using ArcCHECK were not fully elucidated. Before clinical implementation of ArcCHECK for IMRT and VMAT plans, qualified medical physicists should familiarize themselves with its characteristics against dose calculation algorithms.

The purpose of this study was to compare measured dose distributions and calculated ones in $D_{w}$ and $D_{m}$ in simple, patient-specific IMRT and VMAT plans using ArcCHECK with the fixed phantom density recommended by the vendor.

\section{Materials and Methods}

\subsection{ArcCHECK Device}

ArcCHECK is a cylindrical polymethylmethacrylate (PMMA) phantom with a 3D array of 1386 diode detectors with a 1-cm detector spacing. ArcCHECK has an outer diameter of $26.6 \mathrm{~cm}$ and an inner-hole diameter of $15.1 \mathrm{~cm}$, with the curved plane of diodes at a distance of $10.4 \mathrm{~cm}$ from the center. The detectors spiral down the cylinder length to increase the spatial sampling rate and reduce the detector overlap from the beam's eye view. The active detector size is $0.8 \mathrm{~mm} \times$ $0.8 \mathrm{~mm}$. ArcCHECK measures the dose data in $50-\mathrm{ms}$ intervals, saves all the measurement data as a function of time, and measures both relative and absolute doses.

\subsection{Absolute Dose Calibration}

A water tank (WP1D Water Phantom; IBA Dosimetry GmbH, Germany) was used to measure the absolute dose at a calibration depth of $3.3 \mathrm{~cm}$ according to the guidelines provided by Sun Nuclear Corporation (SNC) [25]. The physical distance from the top surface of the ArcCHECK to the diode detectors was 2.85 $\mathrm{cm}$, which corresponds to a water-equivalent depth of $3.3 \mathrm{~cm}$, as described above.

Before measuring the dose at the calibration depth of $3.3 \mathrm{~cm}$ in water, the dose per monitor unit (D/MU) was measured using the ionization chamber (PTW30013; PTW Co., Freiburg, Germany) annually calibrated by the Japan calibration service system. The chamber was irradiated perpendicular to the beam axis with a radiation field size of $10 \times 10 \mathrm{~cm}^{2}$ at a depth of $10 \mathrm{~cm}$ in water. The center of the ionization chamber was at a source-axis-distance of $100 \mathrm{~cm}$. The dose delivered at the given depth was calculated by multiplying the delivered monitor unit, $\mathrm{D} / \mathrm{MU}$, and the tissue maximum ratio. Thereafter, the doses were measured at a depth of $3.3 \mathrm{~cm}$ and a source-surface distance of $86.3 \mathrm{~cm}$ in water, 
according to SNC instructions [25]. The effective point of the measurement of the ionization chamber was located at the measuring depth. The absolute dose was corrected by D/MU and a displacement correction factor.

\subsection{Absolute Dose Calibration}

Measurements were performed on four treatment machines, including a Clinac iX (Varian Medical Systems, Palo Alto, CA), a TrueBeam STx (Varian), a Vero (Mitsubishi Heavy Industries [MHI], Ltd., Tokyo, Japan) at Kyoto University Hospital, and a TrueBeam (Varian) at Osaka Red Cross Hospital. A 120-leaf Millennium MLC was mounted on the Clinac iX and TrueBeam.

Treatment plans were designed on Eclipse (version 11; Varian) for Varian treatment machines, and iPLAN RT Dose (version 4.5.3; Brainlab AG, Feldkirchen, Germany). AXB (version 11.0.31), with a $2.5-\mathrm{mm}$ grid resolution for Eclipse, and XVMC (version 4.5.3), with a $2.0-\mathrm{mm}$ grid resolution and $2 \%$ dose variance for iPLAN, were used for dose calculation. Table couch structures were used in the calculations. The treatment planning information is summarized in Table 1 .

\subsection{ArcCHECK Phantom-Mass-Density}

The computed tomography (CT) dataset of the ArcCHECK was provided from SNC for dose calculations. The user manual recommended that the ArcCHECK phantom-mass-density should be assigned a density close to that of the PMMA material for the treatment planning system (TPS) dose calculation [25]. The recommended density value of $1.18 \mathrm{~g} / \mathrm{cm}^{3}$ for LBTE- and MC-based dose calculation algorithms (e.g., AXB and XVMC), was assigned to the ArcCHECK on CT images. The density value of $1.18 \mathrm{~g} / \mathrm{cm}^{3}$ for AXB and XVMC was the combination of cartilage and bone density in TPS.

Table 1. Treatment planning information.

\begin{tabular}{|c|c|c|c|c|}
\hline & \multicolumn{3}{|c|}{ Kyoto University Hospital } & Osaka Red Cross \\
\hline $\begin{array}{c}\text { Treatment } \\
\text { machine }\end{array}$ & Clinac iX & TrueBeam STx & Vero & TrueBeam \\
\hline MLC & $\begin{array}{c}\text { Millennium } 120 \\
\text { MLC }\end{array}$ & HD120 MLC & $\begin{array}{c}\text { MHI MLC } \\
60\end{array}$ & $\begin{array}{c}\text { Millennium } 120 \\
\text { MLC }\end{array}$ \\
\hline Energy & $6,15-\mathrm{MV}$ & $\begin{array}{c}6,10,15, \\
\text { 10FFF-MV }\end{array}$ & 6-MV & $6,10-\mathrm{MV}$ \\
\hline $\begin{array}{c}\text { Dose } \\
\text { calculation }\end{array}$ & $\mathrm{AXB}$ & $\mathrm{AXB}$ & XVMC & $\mathrm{AXB}$ \\
\hline $\begin{array}{c}\text { Calculation } \\
\text { grid size }\end{array}$ & $2.5 \mathrm{~mm}$ & $2.5 \mathrm{~mm}$ & $2 \mathrm{~mm}$ & $2.5 \mathrm{~mm}$ \\
\hline
\end{tabular}

Abbreviations: MLC, multi-leaf collimators; HD, high-definition; AXB, Acuros XB; XVMC, X-ray Voxel Monte Carlo; FFF, flattening filter free. 


\subsection{Dosimetric Evaluation for Simple Plans}

A total of 45 simple plans, including the 1-field anterior-posterior direction (1F), the 3-field anterior and left-right direction $(3 \mathrm{~F})$, the 4 -field anterior-posterior and left-right direction $(4 \mathrm{~F})$, a half-arc delivery (half-arc) from $270^{\circ}$ to $90^{\circ}$ gantry angle under clockwise rotation, and a full-arc delivery (full-arc) from $181^{\circ}$ to $179^{\circ}$ gantry angle under clockwise rotation, were assessed. A field size of $10 \times 10$ $\mathrm{cm}^{2}$ was used for all simple plans. The dose distributions were assessed quantitatively by gamma analysis with a $3 \%$ normalized global dose error and a 3-mm DTA threshold $(\gamma 3 \% \mathrm{G} / 3 \mathrm{~mm})$ criterion using SNC software (ver. 6.6) for all plans.

\subsection{Dosimetric Evaluation for Patient-Specific Plans}

A total of 96 patients were assessed retrospectively in this study. Of them, QA for 76 plans was conducted at Kyoto University Hospital and 20 at Osaka Red Cross Hospital. Eighty-five patients were irradiated with VMAT by Varian machines; static IMRT by Vero was used for the remaining 11 patients. This study was approved by institutional review board. A summary of treatment machines, energy, delivery technique, and representative disease site is given in Table 2.

Table 2. Summary of treatment machines, energy, and delivery technique.

\begin{tabular}{|c|c|c|c|c|}
\hline & & $\begin{array}{l}\text { Delivery } \\
\text { technique }\end{array}$ & Representative disease site & $\begin{array}{c}\text { \# of } \\
\text { patients }\end{array}$ \\
\hline \multirow[t]{8}{*}{ KUHP } & & & & 76 \\
\hline & Clinac iX (6-MV) & VMAT & $\begin{array}{l}\text { Head and neck, esophagus, } \\
\text { spine, brain, lung, etc. }\end{array}$ & 9 \\
\hline & Clinac iX (15-MV) & VMAT & $\begin{array}{l}\text { Pelvis, prostate, cervix, } \\
\text { pancreas, rectal, etc. }\end{array}$ & 9 \\
\hline & $\begin{array}{c}\text { TrueBeam STx } \\
(6-M V)\end{array}$ & VMAT & $\begin{array}{l}\text { Head and neck, brain, } \\
\text { esophagus, lung. }\end{array}$ & 10 \\
\hline & $\begin{array}{l}\text { TrueBeam STx } \\
(10-M V)\end{array}$ & VMAT & $\begin{array}{l}\text { Pelvis, prostate, } \\
\text { rectal, pancreas. }\end{array}$ & 6 \\
\hline & $\begin{array}{c}\text { TrueBeam STx } \\
(10 \text { FFF-MV) }\end{array}$ & VMAT & $\begin{array}{l}\text { Pancreas, lymph node, } \\
\text { kidney. }\end{array}$ & 15 \\
\hline & $\begin{array}{l}\text { TrueBeam STx } \\
(15-M V)\end{array}$ & VMAT & $\begin{array}{l}\text { Prostate, pelvis, anal, } \\
\text { pancreas. }\end{array}$ & 16 \\
\hline & Vero $(6-M V)$ & Static IMRT & Prostate, pancreas. & 11 \\
\hline \multirow[t]{3}{*}{ ORC } & & & & 20 \\
\hline & TrueBeam (6-MV) & VMAT & $\begin{array}{l}\text { Esophagus, cervix, lung, } \\
\text { head and neck, spine, etc. }\end{array}$ & 10 \\
\hline & TrueBeam (10-MV) & VMAT & $\begin{array}{l}\text { Prostate, pancreas, lung, } \\
\text { anal, esophagus. }\end{array}$ & 10 \\
\hline
\end{tabular}

Abbreviations: KUHP, Kyoto University Hospital; ORC, Osaka Red Cross Hospital; VMAT, volumetric modulated arc therapy; IMRT, intensity modulated radiotherapy; FFF, flattening filter free. 
Gamma analysis was used to evaluate patient-specific QA. The gamma index at $\gamma 3 \% \mathrm{G} / 3 \mathrm{~mm}$ was assessed in $D_{w}$ and $D_{m}$. As subgroup analyses, dose distributions due to differences in energies, delivery techniques, and treatment machines were also assessed in $D_{W}$ and $D_{m}$.

The paired Wilcoxon signed-rank test explored the change in $\gamma 3 \% \mathrm{G} / 3 \mathrm{~mm}$ between $D_{w}$ and $D_{m}$. Data were statistically analyzed using JMP Pro 11 software (SAS Institute, Inc., Cary, NC, USA). The level of significance for all tests was set at a p-value of 0.05 .

\section{Results}

\subsection{Absolute Dose Calibration}

The differences between the measured dose at a depth of $2.85 \mathrm{~cm}$ in ArcCHECK and the calculated dose at a depth of $3.3 \mathrm{~cm}$ in the water phantom were within $\pm 1.0 \%$ for any combination in $D_{w}$ and $D_{m}$.

\subsection{Dosimetric Evaluation for Simple Plans}

A summary of the $\gamma 3 \% \mathrm{G} / 3 \mathrm{~mm}$ for simple plans is presented in Figure 1. The median values of $\gamma 3 \% \mathrm{G} / 3 \mathrm{~mm}$ for all simple plans, including $1 \mathrm{~F}, 3 \mathrm{~F}, 4 \mathrm{~F}$, half-arc, and full-arc, in $D_{w}$ and $D_{m}$ were $98.1 \%$ (range, $75.2 \%-100 \%$ ) and $95.5 \%$ (range, $23.7 \%-100 \%$ ), respectively. The median pass rates of $\gamma 3 \% \mathrm{G} / 3 \mathrm{~mm}$ for all simple plans in $D_{w}$ were significantly higher than those in $D_{m}(\mathrm{p}<0.01)$.

\subsection{Dosimetric Evaluation for Patient-Specific Plans}

The pass rates of $\gamma 3 \% \mathrm{G} / 3 \mathrm{~mm}$ in $D_{w}$ were significantly higher than those in $D_{m}$.

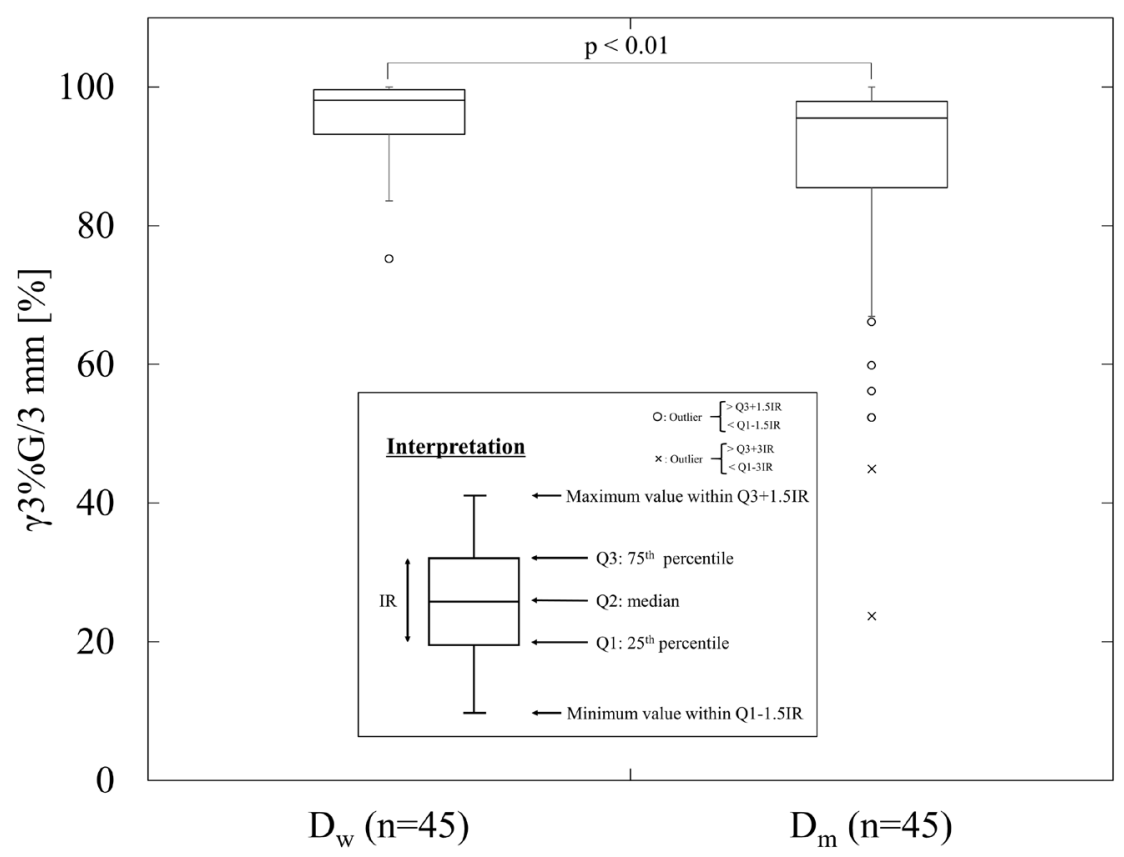

Figure 1. Boxplot of $\gamma 3 \% \mathrm{G} / 3 \mathrm{~mm}$ in dose-to-water $\left(D_{w}\right)$ and dose-to-medium $\left(D_{m}\right)$ for simple plans. The $\gamma 3 \% \mathrm{G} / 3 \mathrm{~mm}$ in $D_{w}$ was significantly higher than those in $D_{m}(\mathrm{p}<0.01)$. 
Figure 2 shows a boxplot of $\gamma 3 \% \mathrm{G} / 3 \mathrm{~mm}$ for patient-specific plans. The median pass rates of $\gamma 3 \% \mathrm{G} / 3 \mathrm{~mm}$ in $D_{w}$ and $D_{m}$ were $98.6 \%$ (range, $90.1 \%-100 \%$ ) and 90.5\% (range, $38.5 \%$ - 97.2\%), respectively. Figure 3 shows boxplots of $\gamma 3 \% \mathrm{G} /$ $3 \mathrm{~mm}$ under various energies, delivery techniques, and treatment machines. The results also showed that all $\gamma 3 \% \mathrm{G} / 3 \mathrm{~mm}$ in $D_{w}$ were significantly higher than those in $D_{m}(\mathrm{p}<0.01)$. Figure 4 shows an example of dose profiles of patient-specific QA with the 10FFF-MV X-ray from the TrueBeam STx. The maximum dose difference between measurement and calculation was $-0.8 \%$ and $3.5 \%$ in $D_{w}$ and $D_{m}$, respectively. The doses calculated in $D_{w}$ were $4.2 \%$ systematically higher than those in $D_{m}$.

\section{Discussion}

The present study is the first reported to assess the comparison between measured dose distributions and calculated ones in $D_{w}$ and $D_{m}$ using ArcCHECK for various plans. We found that the median pass rates of $\gamma 3 \% \mathrm{G} / 3 \mathrm{~mm}$ for all simple plans in $D_{w}$ were significantly higher than those in $D_{m}$ (Figure 1). In addition, the dose distribution calculated in $D_{w}$ agreed with the measured one to a high level of significance, compared with those in $D_{m}$ (Figure 2). AAPM TG 119 reported that the average percentage of $\gamma 3 \% \mathrm{G} / 3 \mathrm{~mm}$ for IMRT was $96.3 \% \pm 4.4 \%$ (mean $\pm 2 \sigma$ [ $\sigma$, standard deviation]) [26]. In addition, several studies have reported pass rates of around 95\% with $\gamma 3 \% \mathrm{G} / 3 \mathrm{~mm}$ using ArcCHECK [18]-[24] [27]. In this study, the median pass rate of $\gamma 3 \% \mathrm{G} / 3 \mathrm{~mm}$ was $98.6 \%$ in $D_{w}$, which is supported by previous reports.

Worldwide, the traceability of QA dosimetry is established in water; therefore, dose calibration protocols for treatment machines and treatment planning

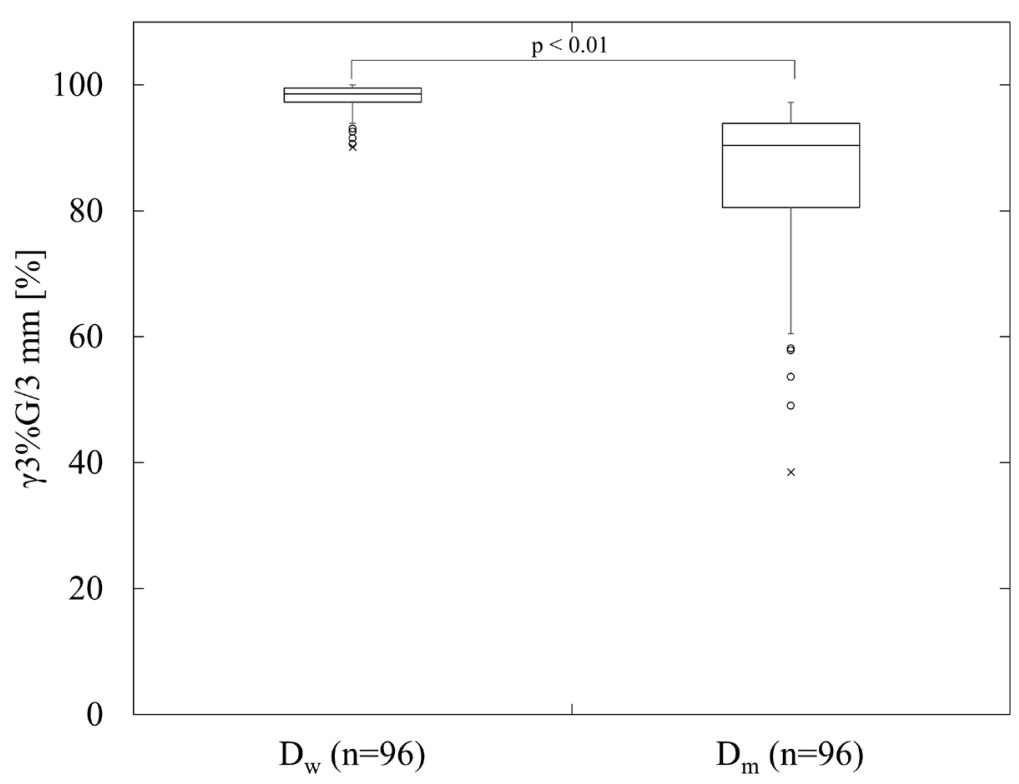

Figure 2. Boxplot of $\gamma 3 \% \mathrm{G} / 3 \mathrm{~mm}$ in dose-to-water $\left(D_{w}\right)$ and dose-to-medium $\left(D_{m}\right)$ for patient-specific plans. The $\gamma 3 \% \mathrm{G} / 3 \mathrm{~mm}$ in $D_{w}$ was significantly higher than those in $D_{m}(\mathrm{p}<0.01)$. 


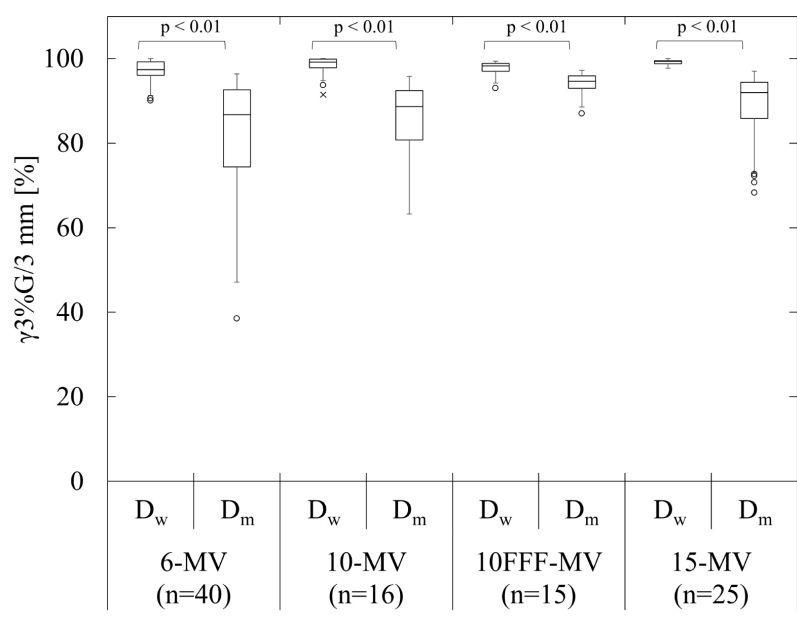

(a)

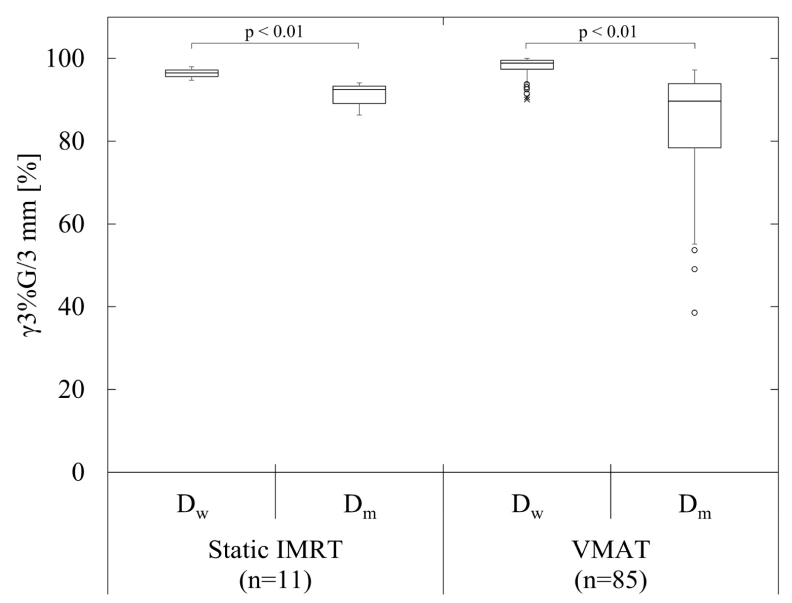

(b)

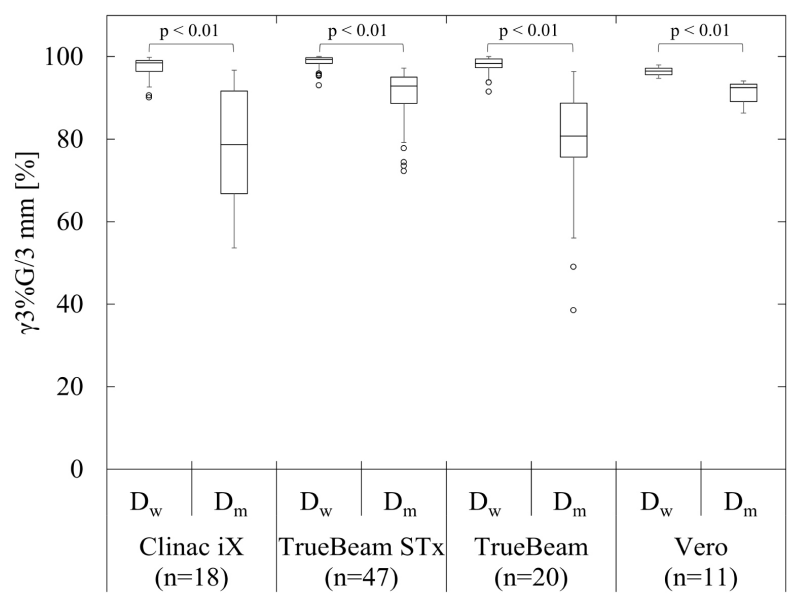

(c)

Figure 3. Boxplots of $\gamma 3 \% \mathrm{G} / 3 \mathrm{~mm}$ under various (a) energies, (b) delivery techniques, and (c) treatment machines in dose-to-water $\left(D_{w}\right)$ and dose-to-medium $\left(D_{m}\right)$. The legend for the box plots is described in Figure 2. The results also showed that all $\gamma 3 \% \mathrm{G} / 3 \mathrm{~mm}$ in $D_{W}$ were significantly higher than those in $D_{m}(\mathrm{p}<0.01)$. 


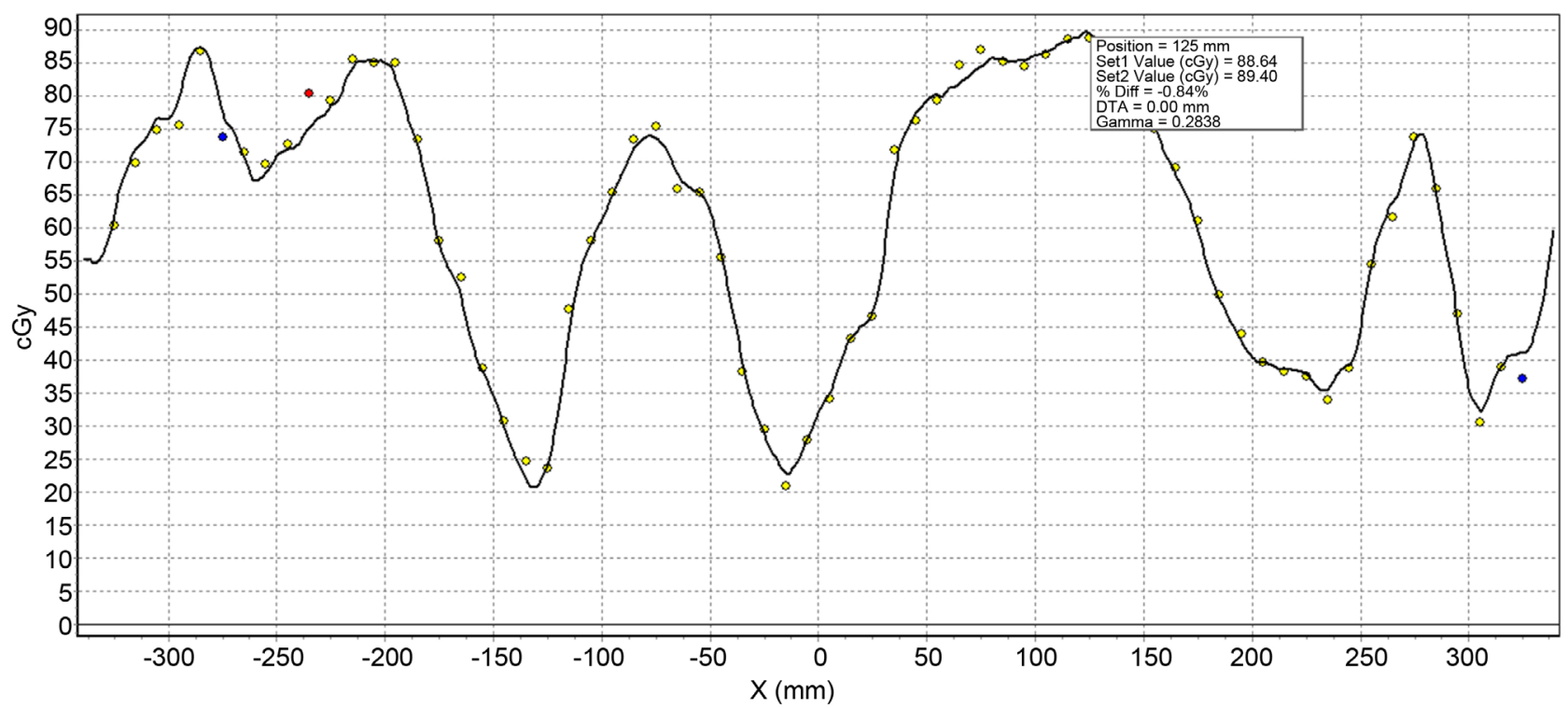

(a)

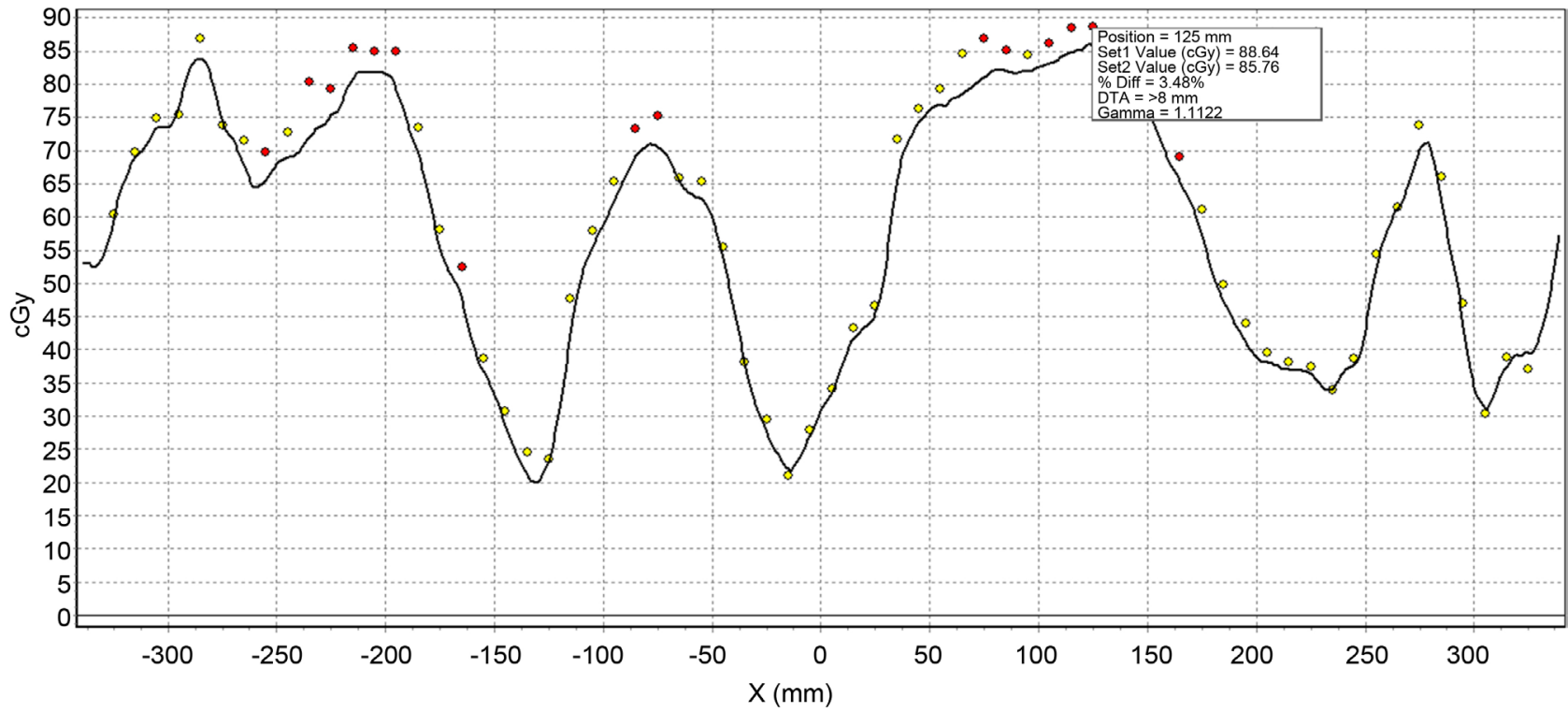

(b)

Figure 4. Dose profiles of patient-specific QA with 10FFF-MV X-ray from TrueBeam STx. (a) dose-to-water ( $\left.D_{w}\right)$ and (b) dose-to-medium $\left(D_{m}\right)$. Dots show measurement values. The yellow, red, and blue dots indicate agreement, higher, and lower doses compared with the calculated dose distribution in $D_{w}$ and $D_{m}$ using $\gamma 3 \% \mathrm{G} / 3 \mathrm{~mm}$ criteria, respectively. The dose of the representative point in box information shows the measured dose and calculated dose. The doses calculated in $D_{w}$ were $4.2 \%$ systematically higher than those in $D_{m}$.

systems should be water-based. The SNC user manual recommends that the ArcCHECK dose calibration be performed in water 25. In this study, the differences between the measured dose at a depth of $2.85 \mathrm{~cm}$ in ArcCHECK and the calculated dose at a depth of $3.3 \mathrm{~cm}$ in the water phantom were within $\pm 1.0 \%$. Therefore, the assigned absolute dose to ArcCHECK was clinically acceptable from the viewpoint of traceability.

The conversion of $D_{m}$ to $D_{W}$ or vice versa requires the application of Bragg- 
Gray cavity theory; $D_{w}=D_{m}(\bar{S} / \rho)_{m}^{w}$, where $(\bar{S} / \rho)_{m}^{w}$ is the unrestricted water-to-medium mass collision stopping power averaged over the energy spectra of primary electrons at the point of interest [8] [13] [17]. The term $(\bar{S} / \rho)_{m}^{w}$ refers to the energy loss by electrons per unit path length of a material, which indicates that the amount of scattered photons and secondary electrons is dependent on $(\bar{S} / \rho)_{m}^{w}$. When dose distributions are calculated in $D_{w}$, the behavior of the energy loss by electrons per unit path length for PMMA might be identical to those for AAA and CCCS [17]. Therefore, the $\gamma 3 \% \mathrm{G} / 3 \mathrm{~mm}$ of our results calculated in $D_{w}$ were consistent with those calculated in AAA and CCCS in the previous studies [18]-[24].

The possibility, at first provided by Monte Carlo simulation, of reporting the dose as $D_{m}$, has still become a subject of controversy in medical physics community [11] [14]. For tissues with densities around $1.00 \mathrm{~g} / \mathrm{cm}^{3}$, the dose difference between $D_{w}$ and $D_{m}$ for megavoltage photon beams is generally small ( $\left.1 \%-2 \%\right)$ [8]. The clinical impact of switching from $D_{m}$ to $D_{w}$ is not expected to be significant, mainly because most tissues of interest in radiotherapy are similar to water [13] [14]. Actually, the dose distributions are routinely calculated in $D_{m}$ in our institution. Therefore, even though the pass rates of $\gamma 3 \% \mathrm{G} / 3 \mathrm{~mm}$ is low in $D_{m}$ of the ArcCHECK, it does not mean that quality of treatment plans in the patients calculated in $D_{m}$ is poor. When dose distributions are calculated in $D_{m}$ for patient-specific QA for IMRT and VMAT plans, the density value of $1.00 \mathrm{~g} / \mathrm{cm}^{3}$ should be assigned to the ArcCHECK on CT images. Note that, however, this density assignment is not recommended by SNC [25].

The cause of lower $\gamma 3 \% \mathrm{G} / 3 \mathrm{~mm}$ in $\mathrm{D}_{\mathrm{m}}$ may be due to $(\bar{S} / \rho)_{m}^{w}$, which varies with phantom-mass-density. According to Siebers et al., the $(\bar{S} / \rho)_{m}^{w}$ of water-to-PMMA was 1.035, which was almost constant, even at different energies [8]. Therefore, the tendencies of $\gamma 3 \% \mathrm{G} / 3 \mathrm{~mm}$ between $D_{w}$ and $D_{m}$ were similar under various energies [Figure $3(\mathrm{a})$ ], and systematic declines in absolute dose were observed in $D_{m}$ due to $(\bar{S} / \rho)_{m}^{w}$ (Figure 4). Similar tendencies were also seen under various delivery techniques and treatment machines [Figure 3(b) and Figure 3(c)]. From our results and the rationale discussed in this study, our approach may be clinically reasonable.

Disease heterogeneity, included in this study, and characteristics of $\gamma 3 \% \mathrm{G} / 3 \mathrm{~mm}$ would be causes of large variations between the results in $D_{w}$ and those in Dm. As shown in the results of the 10FFF-MV X-ray in Figure 3(a) and Vero in Figure $3(\mathrm{c})$, the variations were almost the same between $D_{w}$ and $\mathrm{D}_{\mathrm{m}}$ due to disease homogeneity. However, larger variations were observed for other results, which would be derived from the characteristics of $\gamma 3 \% \mathrm{G} / 3 \mathrm{~mm}$. The concept of the gamma evaluation method combines the dose difference criterion with a DTA criterion for each point of interest [28]. If a global dose difference of $<\mathrm{D} \%$ and/or DTA of $<\mathrm{d}-\mathrm{mm}$ is observed, the gamma value at $\mathrm{D} \% \mathrm{G} / \mathrm{d}-\mathrm{mm}$ passes at the point of interest. In this study, local $\gamma$ failure points in the high dose regions were observed (Figure 4); however, $\gamma 3 \% \mathrm{G} / 3 \mathrm{~mm}$ passed in the lower dose re- 
gions regardless of the dose level decrease in $D_{m}$. At these points, the DTA would be within $3 \mathrm{~mm}$ although the dose differences were greater than $3 \%$. Consequently, the characteristics of $\gamma 3 \% \mathrm{G} / 3 \mathrm{~mm}$ were notable for subgroups including various diseases, which may lead to large variations in $\gamma 3 \% \mathrm{G} / 3 \mathrm{~mm}$ calculated in $D_{m}$.

\section{Conclusion}

This study demonstrated that measured dose distributions and calculated ones in $D_{w}$ agreed well with each other, and showed a greater agreement than those in $D_{m}$ for a range of energies, delivery techniques, and treatment machines from different institutions. From the viewpoint of the rationale of dosimetry, $D_{w}$ shows better agreement with measured dose distribution when using the fixed phantom density recommended by the vendor.

\section{Acknowledgements}

We acknowledge financial support by a Grant-in-Aid for Scientific Research from the Ministry of Education, Culture, Sports, Science, and Technology of Japan (Grant 25253078).

\section{References}

[1] Nutting, C.M., Morden, J.P., Harrington, K.J., et al. (2011) Parotid-Sparing Intensity Modulated versus Conventional Radiotherapy in Head and Neck Cancer (PARSPORT): A Phase 3 Multicentre Randomised Controlled Trial. The Lancet Oncology, 12, 127-136. https://doi.org/10.1016/S1470-2045(10)70290-4

[2] Wolff, D., Stieler, F., Welzel, G., et al. (2009) Volumetric Modulated Arc Therapy (VMAT) vs. Serial Tomotherapy, Step-and-Shoot IMRT and 3D-Conformal RT for Treatment of Prostate Cancer. Radiotherapy and Oncology, 93, 226-233. https://doi.org/10.1016/j.radonc.2009.08.011

[3] Kry, S.F., Molineu, A., Kerns, J.R., et al. (2014) Institutional Patient-Specific IMRT QA Does Not Predict Unacceptable Plan Delivery. International Journal of Radiation Oncology ${ }^{*}$ Biology ${ }^{*}$ Physics, 90, 1195-1201. https://doi.org/10.1016/j.ijrobp.2014.08.334

[4] Failla, G., Wareing, T., Archambault, Y., et al. (2010) Acuros ${ }^{\circledast}$ XB Advanced Dose Calculation for the Eclipse ${ }^{\mathrm{TM}}$ Treatment Planning System [White Paper].

[5] Han, T., Mikell, J.K., Salehpour, M.R., et al. (2011) Dosimetric Comparison of Acuros XB Deterministic Radiation Transport Method with Monte Carlo and Model-Based Convolution Methods in Heterogeneous Media. Medical Physics, 38, 2651 2664. https://doi.org/10.1118/1.3582690

[6] Tsuruta, Y., Nakata, M., Nakamura, M., et al. (2014) Dosimetric Comparison of Acuros XB, AAA, and XVMC in Stereotactic Body Radiotherapy for Lung Cancer. Medical Physics, 41, 081715. https://doi.org/10.1118/1.4890592

[7] Lloyd, S.A.M. and Ansbacher, W. (2013) Evaluation of an Analytic Linear Boltzmann Transport Equation Solver for High-Density Inhomogeneities. Medical Physics, 40, 117072.

[8] Siebers, J.V., Keall, P.J., Nahum, A.E., et al. (2000) Converting Absorbed Dose to Medium to Absorbed Dose to Water for Monte Carlo Based Photon Beam Dose 
Calculations. Physics in Medicine \& Biology, 45, 983-995.

https://doi.org/10.1088/0031-9155/45/4/313

[9] Han, T., Followill, D., Mikell, J., et al. (2013) Dosimetric Impact of Acuros XB Deterministic Radiation Transport Algorithm for Heterogeneous Dose Calculation in Lung Cancer. Medical Physics, 40, 051710. https://doi.org/10.1118/1.4802216

[10] Zhen, H., Hrycushko, B., Lee, H., et al. (2015) Dosimetric Comparison of Acuros $\mathrm{XB}$ with Collapsed Cone Convolution/Superposition and Anisotropic Analytic Algorithm for Stereotactic Ablative Radiotherapy of Thoracic Spinal Metastases. Journal of Applied Clinical Medical Physics, 16, 181-192. https://doi.org/10.1120/jacmp.v16i4.5493

[11] Mampuya, W.A., Nakamura, M., Hirose, Y., et al. (2016) Difference in Dose-Volumetric Data between the Analytical Anisotropic Algorithm, the Dose-To-Medium, and the Dose-To-Water Reporting Modes of the Acuros XB for Lung Stereotactic Body Radiation Therapy. Journal of Applied Clinical Medical Physics, 17, 341-347. https://doi.org/10.1120/jacmp.v17i5.6338

[12] Kan, M.W.K., Leung, L.H.T., So, R.W.K., et al. (2013) Experimental Verification of the Acuros XB and AAA Dose Calculation Adjacent to Heterogeneous Media for IMRT and RapidArc of Nasopharygeal Carcinoma. Medical Physics, 40, 031714. https://doi.org/10.1118/1.4792308

[13] Chetty, I.J., Curran, B., Cygler, J.E., et al. (2007) Report of the AAPM Task Group No. 105: Issues Associated with Clinical Implementation of Monte Carlo-Based Photon and Electron External Beam Treatment Planning. Medical Physics, 34, 4818-4853. https://doi.org/10.1118/1.2795842

[14] Liu, H.H., Keall, P. and Hendee, W.R. (2002) $D_{m}$ Rather than $D_{w}$ Should Be Used in Monte Carlo Treatment Planning. Medical Physics, 29, 922-924. https://doi.org/10.1118/1.1473137

[15] Dogan, N., Siebers, J.V., Keall, P.J. (2006) Clinical Comparison of Head and Neck and Prostate IMRT Plans Using Absorbed Dose to Medium and Absorbed Dose to Water. Physics in Medicine \& Biology, 51, 4967-4980. https://doi.org/10.1088/0031-9155/51/19/015

[16] Usmani, M.N., Masai, N., Oh, R.-J., et al. (2014) Comparison of Absorbed Dose to Medium and Absorbed Dose to Water for Spine IMRT Plans Using a Commercial Monte Carlo Treatment Planning System. International Journal of Medical Physics, Clinical Engineering and Radiation Oncology, 3, 60-66.

https://doi.org/10.4236/ijmpcero.2014.31010

[17] Andreo, P. C. (2015) Dose to "water-like" Media or Dose to Tissue in MV Photons Radiotherapy Treatment Planning: Still a Matter of Debate. Physics in Medicine \& Biology, 60, 309-337. https://doi.org/10.1088/0031-9155/60/1/309

[18] Létourneau, D., Publicover, J., Kozelka, J., et al. (2009) Novel Dosimetric Phantom for Quality Assurance of Volumetric Modulated Arc Therapy. Medical Physics, 36, 1813-1821. https://doi.org/10.1118/1.3117563

[19] Feygelman, V., Zhang, G., Stevens, C., et al. (2011) Evaluation of a New VMAT QA Device, or the "X" and "O" Array Geometries. Journal of Applied Clinical Medical Physics, 12, 146-168. https://doi.org/10.1120/jacmp.v12i2.3346

[20] Nelms, B.E., Chan, M.F., Jarry, G., et al. (2013) Evaluating IMRT and VMAT Dose Accuracy: Practical Examples of Failure to Detect Systematic Errors When Applying a Commonly Used Metric and Action Levels. Medical Physics, 40, 111722. https://doi.org/10.1118/1.4826166

[21] Li, G., Zhang, Y., Jiang, X., et al. (2013) Evaluation of the ArcCHECK QA System 
for IMRT and VMAT Verification. Physica Medica: European Journal of Medical Physics, 29, 295-303. https://doi.org/10.1016/j.ejmp.2012.04.005

[22] Chaswal, V., Weldon, M., Gupta, N., et al. (2014) Commissioning and Comprehensive Evaluation of the ArcCHECK Cylindrical Diode Array for VMAT Pretreatment Delivery QA. Journal of Applied Clinical Medical Physics, 15, 212-225.

https://doi.org/10.1120/jacmp.v15i4.4832

[23] Aristophanous, M., Suh, Y., Chi, P.C., et al. (2016) Initial Clinical Experience with ArcCHECK for IMRT/VMAT QA. Journal of Applied Clinical Medical Physics, 17, 20-33. https://doi.org/10.1120/jacmp.v17i5.6118

[24] Lin, M.H., Koren, S., Veltchev, I., et al. (2013) Measurement Comparison and Monte Carlo Analysis for Volumetric-Modulated Arc Therapy (VMAT) Delivery Verification Using the ArcCHECK Dosimetry System. Journal of Applied Clinical Medical Physics, 14, 220-233. https://doi.org/10.1120/jacmp.v14i2.3929

[25] Sun Nuclear Corporation (2014) SNC Patient Reference Guide. ArcCHECK Density, Sun Nuclear Corporation, Melbourne, FL, 1-8.

[26] Ezzell, G.A., Burmeister, J.W., Dogan, N., et al. (2009) IMRT Commissioning: Multiple Institution Planning and Dosimetry Comparisons, a Report from AAPM Task Group 119. Medical Physics, 36, 5359-5373.

https://doi.org/10.1118/1.3238104

[27] Eaton, D.J., Tyler, J., Backshall, A., et al. (2017) An External Dosimetry Audit Programme to Credential Static and Rotational IMRT Delivery for Clinical Trials Quality Assurance. Physica Medica: European Journal of Medical Physics, 35, 25-30. https://doi.org/10.1016/j.ejmp.2017.02.012

[28] Low, D.A., Harms, W.B., Mutic, S., et al. (1998) A Technique for the Quantitative Evaluation of Dose Distributions. Medical Physics, 25, 656-661.

https://doi.org/10.1118/1.598248 